\title{
Politique énergétique : aspects stratégiques de la question des approvisionnements
}

\author{
Anna Creti ${ }^{(*)}$ \\ Bertrand Villeneuve $e^{(* *)}$
}

Nous traitons des fondements de la fiscalité de l'énergie dans une perspective d'optimisation des approvisionnements auprès de fournisseurs étrangers à la Communauté européenne. Notre modèle permet de distinguer et d'évaluer trois termes formant la taxe optimale: le terme strictement budgétaire, le terme environnemental et le terme stratégique. Les valeurs dépendent de données technologiques (production) et des valeurs des usages (consommation), mais également du rapport de force entre la Communauté et ses fournisseurs. Nous pouvons, à partir des discussions fondées sur les scénarios proposés, suggérer des améliorations de la politique énergétique.

(*) CEA, IDEI et LEERNA, Université de Toulouse I.

E-mail : creti@cict.fr

(**) CEA, IDEI et LEERNA, Université de Toulouse I.

E-mail : bertrand.villeneuve@ cic.fr 
Le problème de la sécurité énergétique en général et celui de la sécurité d'approvisionnement en particulier, ont fait récemment l'objet de plusieurs documents et débats qui témoignent d'un intérêt croissant pour cet aspect classique de la politique énergétique (Bohi et Toman, 1996). Citons le rapport Energy and the challenge of sustainability (World Energy Assessment, 2000) ou le document Energy in Europe-Economic foundations for energy policy (Commission des Communautés Européennes, 1999), et surtout le Livre Vert (Vers une stratégie européenne de la sécurité des approvisionnements, Commission des Communautés Européennes, 2000).

Cette vague de travaux n'est pas sans lien avec la déréglementation des marchés du gaz et de l'électricité qui remet en discussion les principes traditionnels de la sécurité de l'approvisionnement et des réseaux. La thèse du Livre Vert est que, malgré les Directives sur l'ouverture à la concurrence dans ces secteurs, les marchés à eux seuls ne pourront pas soutenir spontanément une offre énergétique suffisamment sûre. Étant donné la faible marge de manœuvre de la Communauté européenne dans le contrôle des pays producteurs, une action en faveur des économies d'énergie, notamment dans les transports et le bâtiment, serait prioritaire. Toutefois, une explication claire de la raison de la défaillance du marché pour les différentes sources d'énergie n'est pas développée ; elle permettrait pourtant de guider les choix politiques de manière beaucoup plus nette.

Dans le cas particulier du gaz, la Commission affirme que même si un marché libre émergeait, en conséquence de la création d'un marché spot et de l'augmentation de la demande de gaz, deux événements seraient pratiquement équiprobables : des prix complètement concurrentiels ou le « cartel du gaz ». Quelle que soit la tendance pour le futur, il faudrait, selon les termes de la Commission, garder le prix du gaz le plus bas possible, et garantir une offre abondante et diversifiée.

L'approche de la Commission reste générale. L'analyse des compromis acceptables entre les divers intérêts est à peine esquissée. La question n'est pas tant d'harmoniser le système de taxation européen que de savoir à quel taux il faut taxer, pour éviter trop de distorsions dans les choix technologiques des utilisateurs d'énergie, que ce soient des entreprises ou des particuliers, compte tenu des objectifs de sécurité énergétique, et des objectifs environnementaux ou simplement budgétaires.

Aussi estimons-nous que les outils conceptuels et analytiques pour étudier le problème n'ont pas beaucoup progressé. Les études dont nous avons connaissance se limitent à calculer, suivant différents scénarios, les réserves prouvées de telle ou telle autre ressource non renouvelable et ensuite des taux de dépendance énergétique (essentiellement, la part d'importations du gaz ou du pétrole sur la consommation totale). Or, cette approche est très sensible à la méthode de calcul des usages des différents sources d'énergie ; la critique est bien connue.

L'objectif de cet article est de faire ressortir le rôle des autorités publiques à l'échelon européen dans l'approvisionnement et la détermination des prix des énergies fossiles en général et du gaz en particulier, qui nous servira d'exemple privilégié. On distingue deux sources d'insécurité dans les approvisionnements :

- les divers moyens employés par les producteurs et les consommateurs pour influencer les prix en leur faveur, provoquant une incertitude stratégique ;

- les événements aléatoires ne trouvant pas leur origine dans les décisions des participants aux marchés énergétiques (chocs de demande, chocs d'offre, pannes et ruptures involontaires, etc.).

Nous traitons des questions proprement stratégiques. Grâce à une modélisation simple du marché du gaz, l'aspect essentiel de l'analyse consiste à détailler les prises de positions stratégiques des parties en présence afin d'apporter des précisions sur les mécanismes de formation du prix et de détermination des quantités échangées.

Les ressortissants de la Communauté européenne achètent du gaz à un pays producteur localisé à l'extérieur de ses frontières et le prix résulte des choix des producteurs et de la politique fiscale. Cette dernière joue un rôle pivot. En effet, si la taxation sert avant tout à financer les dépenses publiques, une autre grande motivation des taxes est la correction d'inefficacité, avec le cas particulièrement important des effets externes (taxation des émissions polluantes). Enfin, la taxe a un effet stratégique vis-à-vis des producteurs de gaz. Pour être plus clair sur ce dernier point, il est nécessaire de préciser le cadre stratégique, ou protocole d'accord, dans lequel des négociations ont lieu. En définitive, c'est l'interaction entre les autorités fiscales défendant l'intérêt européen et le pays producteur qui joue un rôle fondamental dans notre analyse.

Les trois scénarios sont les suivants :

- le scénario normatif (OSR, optimum de second rang). Il permet d'évaluer le surplus maximal qui peut être créé par le marché. Nous donnons un certain nombre de conditions assurant ce résultat : concurrence entre producteurs, position de négociation forte de l'Europe, etc. Il apparaît que ces conditions sont très exigeantes et risqueraient de ne pas être durables si toutefois elles étaient réunies. Nous disposons néanmoins d'une référence pour évaluer tous les autres scénarios, jugés plus réalistes ;

- le scénario où l'Europe est peu active ( $N$, équilibre de Nash d'un jeu simultané). Il s'agit de l'hypothèse selon laquelle l'Europe ne cherche pas à affecter les 
prix par la négociation mais prend le marché comme il vient, en essayant toutefois de bénéficier des ressources fiscales que permet la taxation des énergies et en prenant en compte les externalités environnementales;

- le scénario de renforcement de la position de l'Europe ( $F$, pour force). Dans cette hypothèse, l'Europe cherche à utiliser son pouvoir de marchandage et parvient à imposer un cadre assez strict aux producteurs.

Dans tous les scénarios, nous pouvons mesurer les effets sur la taxation de l'énergie de la motivation budgétaire (obtenir des ressources pour financer l'Etat), de la motivation environnementale (faire internaliser les effets externes de la pollution) et de la motivation proprement stratégique (peser sur le prix TTC pour obtenir un prix HT plus favorable). Le rôle des Etats est donc de coordonner la demande à un niveau qui prenne en considération les effets négligés spontanément par les agents.

La technologie de production est une donnée et peut dans une large mesure être décrite de manière objective. Tel n'est pas le cas du comportement stratégique du producteur. En situation de concurrence, la marge de manœuvre est limitée, puisque le producteur est forcé d'aligner ses prix. Les prix sont toutefois endogènes. Le monopole peut être assimilé à la situation où les producteurs parviennent à se coordonner parfaitement, par exemple en formant un cartel. Cependant, cela ne préjuge en rien de la capacité du monopole à exercer effectivement un pouvoir de marché, car les consommateurs pourraient être encore mieux organisés. D'autre part, les consommateurs peuvent ne pas disposer de pouvoir direct mais leur représentant (la Communauté pour faire court) dispose d'instruments d'influence (la taxation notamment).

Le thème de la taxation des monopoles n'est pas nouveau dans la littérature économique (voir par exemple Bishop, 1968 ; Guesnerie et Laffont, 1978 ; Seade, 1985, et Guesnerie, 1995). Notre contribution s'inspire des travaux de Myles $(1987,1989)$ sur la taxation en concurrence imparfaite. Notre modèle, sans doute plus simple dans la description de l'économie, permet d'identifier les éléments-clés pour la définition d'une politique stratégique au niveau européen, en réponse au problème des approvisionnements énergétiques.

Dans cette étude, les opérateurs de transport et de distribution ne jouent pas de rôle stratégique autonome. Nous supposerons que ces deux secteurs sont concurrentiels. Il est possible d'interpréter cela comme l'hypothèse d'un succès des mesures dessinées par la Directive sur le gaz en vue de promouvoir l'efficacité des réseaux. Cependant, notre formalisation permet de proposer des résultats relatifs à l'évolution des coûts fixes et marginaux du transport et de la distribution permise par la politique européenne. En effet, le coût $c$ (dit de production) peut être vu comme l'ensemble des coûts de mise à disposition (production, transport et distribution). Nous pouvons donc discuter de manière détaillée de l'impact sur l'équilibre du marché d'une éventuelle diminution des coûts marginaux de transport et de distribution.

\section{Plan de l'étude}

La première partie présente les principaux choix de modélisation. La deuxième partie discute des déterminants du niveau de consommation efficace, établissant la référence pour toute la suite. La troisième partie présente les différents scénarios. Les quatrième et cinquième parties présentent les résultats scénario par scénario. Tous les résultats visent à évaluer quelques aspects des marchés énergétiques permettant une comparaison simple mais nuancée. Ce sont en particulier :

- le niveau de consommation de gaz ;

- le niveau des taxes et des prix hors-taxe ;

- le surplus engendré, comparé au maximum théorique ;

- les parts de surplus acquises par la Communauté et les producteurs.

La dernière partie discute et conclut l'ensemble des résultats afin de proposer un petit guide de politique énergétique.

\section{Données fondamentales}

\section{La demande}

La demande de gaz est étudiée sous une forme agrégée notée $q[p]$ où $p$ est le prix. Il est supposé que la demande croisse quand le prix diminue,

(1) $\frac{d p[p]}{d p}<0$

L'objectif des modèles est de traiter $p$ et $q$, ainsi que la taxe $t$, comme des variables endogènes, c'est-à-dire dépendant de données techniques et de décisions stratégiques que nous formalisons.

Nous dérivons la demande d'un programme représentant la maximisation d'une utilité sous contrainte de budget, que nous simplifions en lui donnant la forme quasi-linéaire ci-dessous. Nous sommes alors en mesure d'incorporer le bien-être des consommateurs dans l'objectif des autorités politiques. Cette approche synthétique permet de comparer entre elles deux politiques et permet donc d'orienter la recherche d'un optimum. 
La consommation de gaz $q$ est solution de

(2) $\max _{q} U[q]-p q$

Les différences entre les agents dans leurs dispositions à payer pour les paniers quantités/services ne sont pas représentées ni exploitées ici. Ces différences ne produisent pas un effet négligeable mais il est utile pour comprendre les enjeux stratégiques de se limiter aux effets simples des prix sur le niveau général de la demande.

Afin de pouvoir pousser les calculs assez loin, il est commode d'utiliser une fonction d'utilité puissance :

$$
U[q]=\frac{\tau \gamma}{\gamma-1} q^{\frac{\gamma-1}{\gamma}}, \text { où } \gamma>1
$$

Si les échanges sont nuls, alors le surplus est nul. L'optimisation de la demande donne

(4) $q[p]=\left[\frac{p}{\tau}\right]^{-\gamma}$

L'intérêt de l'expression (4) est multiple : $\tau$ détermine le niveau de la demande tandis que $\gamma$ en représente l'élasticité puisque

(5) $-\frac{p}{q} \frac{d q[p]}{d p}=\gamma$

La spécification de la demande que nous avons choisie a l'avantage de résumer l'élasticité-prix dans le paramètre $\gamma$. Cela nous semble cohérent avec notre objectif d'analyser non seulement le niveau des prix et des taxes dans les différents scénarios proposés, mais aussi les parts de surplus capturées par le gouvernement et les producteurs, ainsi que de développer différents exercices de statique comparative. La fonction de demande linéaire, très commode et souvent utilisée (par exemple Wirl, 1993) présenterait un inconvénient majeur. L'élasticité de la demande varie suivant le point où elle est calculée ; autrement dit, l'élasticité prix n'est pas exogène dans le modèle et peut s'écarter de toute vraisemblance empirique.

Dans la littérature économétrique sur la fonction de demande d'énergie, l'estimation de l'élasticité-prix est l'une des questions les plus controversées (voir Madlener 1996 pour une synthèse). Si après le choc pétrolier des années soixante-dix les modèles ont privilégié l'estimation de l'élasticité de la demande d'énergie au niveau macro-économique, en essayant surtout de comprendre si l'énergie est un complément ou un substitut du capital (Berndt et alii, 1975 ; Griffin, 1977), plus récemment des études détaillées de la demande des différentes sources d'énergie (gaz, électricité et pétrole) ont vu le jour (Woodland, 1993).

La réactivité de la consommation au prix varie beaucoup suivant les études : la spécification du modèle et les techniques d'estimation, mais aussi le niveau d'agrégation des données, la distinction entre clients résidentiels et industriels, la durée des séries temporelles ont un impact certain sur la valeur de l'élasticité.

En limitant notre attention aux études agrégées, qui paraissent plus adaptées à un calibrage du paramètre $\gamma$ dans notre modèle, nous remarquons qu'en général les élasticités de long terme sont plus fortes que celles de court terme (Renou-Massant, 1999) : cela s'explique aisément, car à long terme on peut supposer que les investissements ont été optimisés. L'hypothèse d'une élasticité-prix de long terme assez élevée ne peut pas être écartée, comme le montre pour le secteur gazier européen l'étude de Mathiesen et alii (1987). La moyenne des élasticités-prix estimées à long terme dans les pays considérés (Hollande, France, Belgique, Allemagne, Luxembourg, Italie et Angleterre) dans l'étude s'élève à 0,96 ; 1'Italie, la Belgique et le Luxembourg ont une élasticité supérieure à 1 . Supposer $\gamma>1$ ne paraît pas trop irréaliste si l'on se place dans une perspective de long terme.

\section{L'offre}

Deux types de clarifications doivent être faites dans la modélisation de l'offre :

- la description des coûts de production ;

- le type de comportement des producteurs, notamment les cas extrêmes de comportements concurrentiel ou monopolistique.

\section{Les coûts de production et l'objectif du producteur}

Les coûts de production $C[q]$ seront modélisés sous la forme :

(6) $C[q]=K+c q$

Le coût fixe introduit une forme de contrainte de rationalité du producteur : il est nécessaire pour qu'il soit actif que la combinaison prix-quantité lui permette de recouvrer aussi le coût fixe. Cela introduit une première possibilité de distorsion par rapport à l'égalisation du prix au coût marginal. La forme linéaire peut être vue comme une approximation convenable d'une plus vaste catégorie de fonctions. 
Dans toute la suite, nous supposerons que l'objectif des producteurs est la maximisation du profit, soit la différence entre les recettes brutes et les coûts de production. Le profit s'écrit donc

(7) $\pi=(p-c) q-K$

\section{Comportement stratégique}

Nous étudierons la concurrence parfaite et le monopole selon des modalités précises pour chaque scénario.

\section{Objectif de la Communauté}

L'Europe maximise son surplus et les profits des producteurs ne sont pas comptés puisqu'ils sont réalisés hors Communauté. Cela introduit une différence très importante par rapport à l'étude de la régulation interne de l'Europe, où tout est conservé sur le territoire. Concrètement, la Communauté valorise les moyens d'influencer les prix à son avantage et notamment de limiter les profits, alors que si les profits étaient pris en compte, on retrouverait la problématique de la régulation des monopoles.

Nous l'écrirons dans le cas du gaz (pour une taxe nulle)

(8) $U[q]-p q-\eta q+S-(1+\lambda) R$

L'objectif de la Communauté prend en compte tous les effets internes. Les deux premiers termes recoupent parfaitement les objectifs des consommateurs :

$-U[q]$ : le surplus brut permis par la consommation de gaz;

- $p q$ : le prix à payer aux producteurs donc à soustraire.

Les deux termes suivants sont spécifiques aux questions de politique publique :

$-\eta q$ : les effets externes dus à la consommation de gaz. La valeur est négative, ainsi qu'on l'attend d'impacts environnementaux. Ce terme n'est pas pris en compte dans le choix individuel ${ }^{(1)}$;

$-S$ : le surplus exogène engendré par les activités des gouvernements ;

$-R$ : les revenus fiscaux exogènes nécessaires pour financer les activités des gouvernements ;

$-\lambda$ : le coût des fonds publics.

La règle de Pigou consiste à imposer une taxe égale au dommage marginal ( $\eta$ dans notre modèle) : la taxe permet alors de ramener une économie concurrentielle vers la frontière de Pareto. Toutefois, dans un monde où d'autres distorsions sont présentes, donc dans un contexte de second rang, la règle de Pigou doit être modifiée, car il existe une interaction entre les différents instruments fiscaux existant dans une économie et la taxe sur le bien polluant (Sandmo, 1975). Pour modéliser d'une façon synthétique ces interactions, notre modèle se réfère à la théorie du "double dividende" élaborée par Pearce (1991). Selon cette théorie, une taxe sur le bien polluant a deux effets positifs : elle implique une réduction de la pollution (premier dividende) et dégage une recette fiscale permettant de diminuer d'autres impôts, comme par exemple celle sur le travail (second dividende). Cela diminue globalement les distorsions existant dans l'économie. L'existence d'un second dividende dans la taxation du bien polluant ne fait pas l'unanimité dans la littérature. Néanmoins, comme Goulder (1995) le montre, le second dividende peut effectivement exister à condition que le système fiscal initial soit suffisamment éloigné de l'optimum et qu'il implique une taxation trop faible du bien polluant en question. Pour avoir un second dividende, nous supposons donc $\lambda>0^{(2)}$.

Le coût des fonds publics signifie ici que, à la marge, un Euro perçu sur les biens et services non modélisés coûte en surplus $1+\lambda$ Euro $^{(3)}$. Percevoir $R$ reste efficace dans la mesure où $S-(1+\lambda) R>0$.

Le gaz apparaît comme une source de revenu dont les mérites et les inconvénients sont à comparer avec ceux des autres biens. Une taxe à la consommation unitaire de gaz, notée $t^{(4)}$ permet d'obtenir une partie des ressources destinées à financer $R$, pour un coût qui reste à déterminer. En effet, le produit de la taxe sur le gaz $t q$ permet de réduire d'autant les taxes sur les autres biens, ce qui engendre un terme positif dans le surplus $\lambda$ tq. Cependant, la taxation du gaz est limitée par les distorsions qu'elle crée. À l'optimum (voir la deuxième partie), les distorsions entre la fiscalité du gaz et celle des autres biens s'accordent au sens du découragement de Mirrlees (1976).

Nous pouvons réécrire l'objectif de la Communauté (pour ne garder que les termes variables, nous n'écrivons plus $S-(1+\lambda) R$ dans la suite)

(9) $U[q]-p q-\eta q+\lambda t q$

où $q=q[p+t]$. 


\section{Production efficace}

La recherche du niveau efficace de production fait abstraction de la question du prix et donc de la redistribution du surplus créé par l'échange. Ce niveau étant établi, il est possible de chercher si un certain type d'organisation du marché permet de l'atteindre. Lorsque tel est le cas, la discussion porte sur les facteurs modifiant le partage du surplus entre les parties ; sinon, il reste possible de mesurer les pertes d'efficacité d'un système de marché donné et de proposer des remèdes.

\section{Optimum de premier rang (OPR)}

La quasi-linéarité des objectifs impose que tout optimum de Pareto maximise la somme des objectifs particuliers. Cet optimum est calculé sous l'hypothèse qu'il est possible à la Communauté de contrôler les niveaux de consommation, autrement dit de coordonner parfaitement la demande. On résout

(10) $\max _{q} U[q]-c q-\eta q-K$,

ce qui donne, si $K$ est assez petit pour que l'exploitation engendre un surplus positif à l'optimum,

$$
q^{\mathrm{OPR}}=\left[\frac{c+\eta}{\tau}\right]^{-\gamma}
$$

Seuls les coûts réels (coût de production, coût externe) entrent en jeu. Cet optimum n'est plus envisagé dans la suite.

Optimum de second rang (OSR)

Comment mettre en œuvre une telle production? Les consommateurs ne se voient pas dicter leur consommation mais répondent au prix et à la taxe. Par ailleurs, il est nécessaire de compenser les producteurs de leur coût fixe $K$. Cela impose des transferts de la part de la Communauté, soit par le prix, soit sous forme forfaitaire. En fait, il est généralement optimal de combiner les deux comme le calcul qui suit le montre.

Notons $t$ la taxe qui s'ajoute au coût marginal et $T=t q-K$ le résidu fiscal du gaz, soit le produit de la taxe moins le coût fixe. Il faut résoudre

(12) $\max _{t} U[q[c+t]]-(c+\eta) q+\lambda t q$

Ici, comme dans la suite, nous résolvons la condition de premier ordre (la solution est unique et elle satisfait la condition de second ordre). On obtient où la quantité consommée à l'optimum vaut

(15) $q^{\mathrm{OSR}}=\left[\frac{\gamma(\eta+c(1+\lambda))}{\tau(\gamma+\lambda(\gamma-1))}\right]^{-\gamma}$

et où la taxe comprend

$\frac{\eta \gamma}{\gamma+\lambda(\gamma-1)}$ : un terme Pigouvien, destiné à la correction de l'effet externe. Le cas $\lambda=0$ permet de retrouver l'expression classique net donc l'optimum de premier rang ;

$\frac{c \gamma}{\gamma+\lambda(\gamma-1)}:$ un terme d" "exploitation" fiscale du gaz.

Le prix TTC est donc

$$
c+t^{\mathrm{OSR}}=\frac{\gamma(\eta+c(1+\lambda))}{\gamma+\lambda(\gamma-1)}
$$

Elasticité et coût de fonds publics interviennent dans chacun des termes. La taxe $t$ constitue (pour des valeurs faibles) le moyen le moins coûteux socialement d'obtenir des fonds. Il est donc logique de privilégier ce moyen par rapport à la taxation générale. Si la taxation $t$ produit peu de distorsion (notamment si $K$ est petit ou si l'élasticité de la demande au prix est très faible), alors le gaz est taxé non seulement pour couvrir le coût fixe mais aussi pour financer le budget général de l'État, soit $T>0$. Il devient possible, grâce aux ressources fiscales sur l'énergie, d'alléger la fiscalité générale. Si, au contraire, les distorsions deviennent trop importantes, alors il est optimal de couvrir le coût fixe en faisant appel à la fiscalité générale. On obtient alors $T<0$.

On voit donc que la fiscalité de l'énergie présente trois avantages, les deux premiers étant en fait absolument inséparables dans une vision cohérente des finances publiques :

- elle permet de percevoir des ressources fiscales de manière efficace, en alignant le niveau de distorsion sur ce marché sur la distorsion générale ;

- elle permet de couvrir les coûts fixes de la production (et éventuellement du transport, et de la distribution);

- elle permet d'internaliser les conséquences environnementales (effets externes).

(13) $t^{\mathrm{OSR}}=\frac{\eta \gamma+c \lambda}{\gamma+\lambda(\gamma-1)}$

(14) $T^{\mathrm{OSR}}=t^{\mathrm{OSR}} q^{\mathrm{OSR}}-K$ 


\section{Analyse des politiques énergétiques}

L'étude précédente permet d'évaluer tout le surplus potentiel des marchés énergétiques. Chaque scénario demande donc une évaluation :

- des niveaux de consommation, du prix et de la taxe ;

- du niveau de surplus total engendré, comparé à l'optimum ;

- de la distribution du surplus engendré entre les partenaires (consommateurs/producteurs).

La première section donne quelques résultats utopiques. La deuxième section présente le plan d'étude des différentes dimensions que nous pensons importantes. Les scénarios sont développés et comparés dans les quatrième et cinquième parties.

Quel usage peut-il en être fait ? Aucun scénario n'a pour but de représenter plus particulièrement le réel, mais tous le représentent d'une certaine façon. Il est important de comprendre que les scénarios contiennent tous une part de décision stratégique, autrement dit, en examinant un scénario, nous donnons des indications sur les conséquences d'un choix politique qui nous y placerait. À partir de là, il est possible d'esquisser les orientations politiques les plus favorables.

\section{Solutions coopératives (marchandage)}

L'idée des jeux coopératifs est que toute solution adoptée par les parties est efficace. En effet, partons d'une solution dominée, c'est-à-dire laissant inexploitées des possibilités de surplus. L'une des deux parties peut proposer un arrangement plus efficace assurant à chacune des parties au moins le niveau d'utilité de l'arrangement inefficace précédent. Le surplus additionnel peut être partagé de différentes manières, mais tout le monde étant gagnant, cette possibilité ne devrait pas être inexploitée. Bien entendu la réalité est un peu plus complexe.

La question reste donc de savoir comment le surplus est partagé. Intuitivement, la part de surplus que chaque partie peut réclamer est liée à son pouvoir de nuisance ${ }^{(5)}$. Rubinstein (1982) montre, dans l'exemple caractéristique du partage de gâteau, que la partie la plus patiente (celle pour lequel le coût de report d'un accord est le plus faible, donc pour qui le coût de nuire en sabotant l'accord est relativement plus faible) parvient à capturer une part plus grande. Il est intéressant de remarquer que la guerre d'usure n'a jamais lieu dans ce genre d'analyse, puisque le résultat est efficace ; c'est la menace d'une guerre, dont on peut prévoir le vainqueur, qui permet dès aujourd'hui le partage ${ }^{(6)}$.

Dans les modèles d'application, on résume souvent le partage de surplus par un paramètre. En l'absence de toute transaction, le surplus des deux parties est nul ${ }^{(7)}$. Nous noterons $\mu$ la part de surplus que l'Europe capturera et $1-\mu$ la part capturée par les producteurs. Les quantités produites et consommées étant les mêmes dans toutes les circonstances, comme on l'a vu dans l'étude de l'allocation efficace, la question de la mise en œuvre pratique de l'optimum se pose.

Pour tout coefficient de partage du surplus $\mu$, la taxe à la consommation $t^{\mathrm{OSR}}$ et un transfert forfaitaire entre parties permettent la mise en œuvre de la solution coopérative. Le principe est le suivant. Les consommateurs doivent consommer de manière efficace (soit $q^{\text {OSR }}$ ), ce qui impose le prix TTC $c+$ $t^{\mathrm{OSR}}$. Le niveau de taxe optimal est commandé par l'arbitrage entre taxation de l'énergie et taxation des autres biens (non modélisés, sinon implicitement par le paramètre de coût des fonds publics $\lambda$ ). Il reste à transférer entre parties afin de trouver le partage défini $\operatorname{par} \mu$.

\section{Jeux non coopératifs}

Les solutions coopératives, pour intéressantes qu'elles soient, sont souvent irréalistes. En fait, la guerre d'usure a souvent lieu (dans des formes assez chaotiques) : les producteurs peuvent tenter différentes stratégies de quotas, de discrimination, les consommateurs peuvent décréter un embargo, exiger des contreparties, exercer une pression diplomatique voire militaire. La grande question est celle de la crédibilité des prises de position, puisqu'il ne suffit pas de durcir sa position si l'autre partie en fait autant ou davantage.

Concrètement, les économies connaissent des chocs d'origine interne qui peuvent changer le niveau optimal de production ou de demande. Par exemple, la Russie connaît un besoin chronique de liquidités, ce qui affaiblit sa position dans le marchandage, mais de façon variable dans le temps. L'Algérie subit une guerre civile qui pourrait (ou aurait pu) menacer ses exportations énergétiques. L'Europe connaît quant à elle des chocs de demande, ou d'élasticité, des vagues technologiques, des changements fiscaux, motivés notamment par des problématiques environnementales. Ajoutons que l'Europe n'unit pas ses efforts, ce dont peuvent profiter les producteurs, et qu'elle peut par ailleurs subir de manière variable les chocs de demande des autres régions consommatrices de gaz. Ces phénomènes ne sont pas modélisés mais expliquent les manières imparfaites d'établir un équilibre entre offre et demande.

Nous quittons donc l'approche normative pour aborder l'approche positive. Au lieu, comme nous venons de le faire, de caractériser les optima et de donner les règles de mise en œuvre, nous adopterons dans la suite la méthode d'analyse suivante. Dans tous les cas de figure, il est utile de bien comprendre les différents instruments qui sont ou qui pourraient 
être à la disposition de la Communauté ou des producteurs ; il reste à comprendre que l'usage qui en est fait dépend du rapport de force dans le jeu. L'idée est de représenter le jeu d'une manière à la fois complète (ne pas passer à côté d'instruments simples et puissants) et synthétique (trouver un ensemble réduit d'instrument "purs" susceptibles de recouper tous les instruments réels).

L'article de Wirl (1993) est, à notre connaissance, la première contribution soulignant que la relation entre pays producteurs et pays importateurs d'énergie doit être étudiée en utilisant la notion de comportement stratégique. L'auteur se concentre sur l'analyse du secteur pétrolier et compare le prix imposé par un monopole de production (l'OPEP) quand les effets externes ne sont pas taxés, par rapport au cas où la Communauté européenne impose une taxe. Les parties peuvent jouer un jeu simultané ou séquentiel, et l'analyse du jeu où les producteurs sont en position de force est privilégiée. Dans le travail de Wirl, l'analyse des jeux de Nash et Stackelberg reste "accessoire" par rapport au cœur de l'article, où l'auteur montre que la taxation de l'énergie peut encourager les producteurs à former un cartel, ce qui ensuite se traduit par une augmentation du prix à l'importation. Wirl néglige donc la description des effets non-stratégiques (notamment, la motivation budgétaire des taxes), mais surtout l'analyse de la production efficace, du bien-être des parties et donc des effets distributifs de la taxation, ce qui est par contre crucial dans notre modèle, comme nous le montrons dans les quatrième et cinquième parties.

\section{Scénarios étudiés}

Pour chaque scénario, les quantités, les prix et la distribution du surplus sont analysés. En effet, l'imperfection des instruments peut se présenter de manières très diverses et chaque cas demande une analyse spécifique. Bien entendu, nous établirons des configurations caractéristiques d'une certaine famille afin de couvrir en quelques cas seulement l'essentiel des problèmes.

Dans tous les cas (sauf le scénario concurrentiel), le producteur est un monopole. Les variantes sont organisées selon les axes suivants (voir tableau) :

- l'Europe dispose soit d'une politique fiscale complète, auquel cas la taxation est associée à des possibilités de transferts entre l'Europe et les producteurs, soit d'une politique fiscale limitée, dans laquelle les transferts n'ont pas cours ;
- les actions peuvent être simultanées, auquel cas il n'y a pas de partie leader et les actions de chacun sont simplement "réalistes" face aux choix adverses, ou au contraire, l'une des parties peut imposer son cadre à l'autre, ce qui permet l'écriture d'un jeu séquentiel. Comme on le sait, le leader de Stackelberg (premier joueur) dans cette structure bénéficie d'un grand avantage, mais qui reste à mesurer selon les instruments dont il dispose.

\section{Niveaux des prix et des consommations}

La première section traite de deux scénarios extrêmes, conduisant tous deux à l'efficacité maximale. Les deuxième et troisième sections traitent chacune d'un scénario dans lequel les instruments sont imparfaits.

\section{Deux cas extrêmes}

\section{Scénario OSR : stratégies complètes}

Un jeu non-coopératif peut conduire à une solution efficace. Cependant, cela n'est possible qu'à certaines conditions assez restrictives. En effet, si la Communauté peut à la fois taxer la consommation et transférer (verser une compensation forfaitaire ou faire payer un droit d'entrée au producteur), et si elle dispose de tout le pouvoir de marchandage, alors elle peut garantir l'efficacité. Il lui suffit d'adopter la taxe optimale et de calculer au plus juste le transfert. Le producteur, reconnaissant sa position de faiblesse, fait au mieux dans le cadre imposé, conçu précisément pour qu'il soit acceptable, sinon avantageux.

\section{Scénario CP: la concurrence parfaite}

Nous appelons marché concurrentiel une situation dans laquelle les producteurs sont supposés être "preneurs de prix", autrement dit, ils n'ont pas (individuellement) d'influence sur le prix. Ils ne peuvent que choisir la quantité produite. À partir de là, nous pouvons déduire que le prix HT est égal au coût marginal

(17) $p=c$

Le problème de couverture des coûts fixes se pose, mais il ne peut pas être résolu par le prix HT. Une manière de faire consiste à subventionner, par exemple en versant une somme forfaitaire aux

\begin{tabular}{|c|c|c|c|}
\hline Scénario & Producteurs & Instruments de la Communauté & Type de jeu \\
\hline OSR & Monopole & Taxes et transferts & Séquentiel \\
\hline $\mathrm{CP}$ & Concurrence & Taxes et transferts & Indifférent \\
\hline $\mathrm{N}$ & Monopole & Taxes & Simultané \\
\hline $\mathrm{F}$ & Monopole & Taxes & Séquentiel \\
\hline
\end{tabular}


producteurs participants (la forme que cela peut prendre en pratique restant à déterminer), et à leur faire jouer le jeu concurrentiel.

La difficulté est de faire participer les producteurs, mais s'ils participent, le prix est idéal. Nous retrouvons une idée assez courante, selon laquelle il peut être utile d'entretenir une certaine concurrence par le biais de subventions. Le premier problème de cette méthode est qu'il n'est pas du tout certain que ce principe soit toujours exploité de manière efficace (il est sujet à des manipulations de toutes sortes de la part des administrations et décideurs politiques). Le second problème est que le jeu de la concurrence doit être contrôlé afin d'éviter les collusions entre producteurs. Il est clair que les moyens classiques de lutte contre les pratiques anticoncurrentielles ne peuvent pas s'appliquer dans le cas de la production de gaz, tout simplement parce que les producteurs ne sont pas du ressort administratif de la Communauté.

Dans un contexte de concurrence imparfaite (petit nombre de producteurs interagissant stratégiquement les uns contre les autres, par exemple dans une concurrence à la Cournot, ou reposant sur les avantages de localisation), il est possible, mais moins efficace, d'appliquer une taxe à la consommation et d'en verser le produit aux producteurs. La suite privilégie l'étude des relations bilatérales entre consommateurs et producteurs organisés en monopole.

\section{Scénario $\mathbf{N}$ : des stratégies simplistes}

Les deux parties déterminent simultanément leurs stratégies, chacune raisonnant sur l'hypothèse d'un certain choix ferme de la part de l'autre. Un équilibre de Nash (d'où le symbole $N$ ) est atteint lorsque les prévisions de chacun sont réalisées. Les consommateurs quant à eux ne sont sensibles qu'au prix net $p+t$, cela étant connu de la Communauté et des producteurs. Ce scénario reste assez réaliste et permet, en contrastant ses conséquences à celles des autres, de mesurer ce qui est perdu faute d'une structure d'interaction efficace.

\section{Prix et taxes}

Le producteur maximise son profit, connaissant la réaction des consommateurs au prix TTC. Sa fonction de réaction est donc

(18) $p[t]=\frac{c \gamma+t}{\gamma-1}$

où $t$ est le choix de la Communauté attendu. Cette réponse optimale n'est définie que si $p[t]+t>0$ (le consommateur doit payer un prix TTC strictement positif pour que sa consommation soit définie).
Cette formule comprend deux termes répondant à des logiques distinctes :

$\frac{c \gamma}{\gamma-1}$ correspond au "mark-up" classique du monopole (le prix est fixé au-delà du coût marginal, à un niveau plus élevé pour les élasticités plus faibles);

$\frac{t}{\gamma-1}$ est la réaction à la taxe proprement dite. À prix TTC $(p+t)$ égal, une taxe plus élevée d'un certain montant diminue la recette unitaire du même montant. La seule réaction possible est de surcompenser cette «spoliation» par une hausse du prix TTC, en comptant sur l'élasticité faible pour limiter les pertes de recette.

La Communauté choisit $t$ de manière à maximiser

$$
U[q[p+t]]-(p+t) q+(1+\lambda) t q-\eta q
$$

où $p$ est le choix des producteurs attendu. Ce qui donne la fonction de réaction

$$
t[p]=\frac{\eta \gamma+\lambda p}{\gamma+\lambda(\gamma-1)}
$$

où cette réponse optimale n'est définie que si $t[p]+p>0$. Nous avons également deux termes :

$\frac{\eta \gamma}{\gamma+\lambda(\gamma-1)}$ représente le niveau de taxe nécessaire pour la correction de l'effet externe. D'ailleurs, si $\lambda=0$ (aucune distorsion fiscale), nous retrouvons le niveau pur de taxe Pigouvienne grâce auquel les consommateurs perçoivent, par la taxe, le dommage marginal fait à l'économie. Pour $\lambda>0$ la formule prend en compte le fait que taxer le bien est désirable, mais ne néglige pas l'élasticité. En fait, la tentation de taxer beaucoup est freinée et même contrecarrée par l'effet d'élasticité ;

$\frac{\lambda p}{\gamma+\lambda(\gamma-1)}$ représente la taxation non pas stratégique, mais plutôt adaptative. (On comparera avec la valeur stratégique de la section suivante). Plus le prix est grand et plus l'élasticité apparente de la consommation par rapport à la taxe est faible. En effet

(21) $-\frac{t}{q} \frac{\partial q}{\partial t}=\frac{t \gamma}{p+t}$

est, toutes choses égales d'ailleurs, une fonction décroissante de $p$. 
Un équilibre est une solution du système. On peut dire aussi que c'est un point fixe de l'application qui à une certaine hypothèse $(p, t)$ sur les stratégies des adversaires associe les stratégies $(p[t], t[p])$ des joueurs. Nous trouvons alors

$$
\begin{aligned}
& t^{N}=\frac{c \lambda+\eta(\gamma-1)}{\gamma-1+\lambda(\gamma-2)} \\
& p^{N}=\frac{c(\gamma+\lambda(\gamma-1))+\eta}{\gamma-1+\lambda(\gamma-2)}
\end{aligned}
$$

qui ne sont compatibles avec les restrictions de domaine de définition des réactions que si $p^{N}+t^{N}>0$ donc si $\gamma-1+\lambda(\gamma-2)>0$.

Nous pouvons en déduire que l'équilibre de Nash en stratégies pures n'existe pas pour une élasticité comprise entre 1 et $\frac{1+2 \lambda}{1+\lambda}$. Ce problème d'existence peut être vu comme un défaut supplémentaire de ce scénario, car il ne se pose pas pour les autres.

Il est intéressant d'étudier le niveau relatif de la taxe. Pour cela remarquons que

$$
\frac{t^{N}}{p^{N}}=\frac{\lambda c+\eta(\gamma-1)}{c(\gamma+\lambda(\gamma-1))+\eta}
$$

$$
\begin{gathered}
\text { Graphique } 1 \text { : ratio } \frac{t^{N}}{p^{N}} \text { en fonction de } \gamma \\
(\lambda=0,33 \text { et } \eta=0)
\end{gathered}
$$

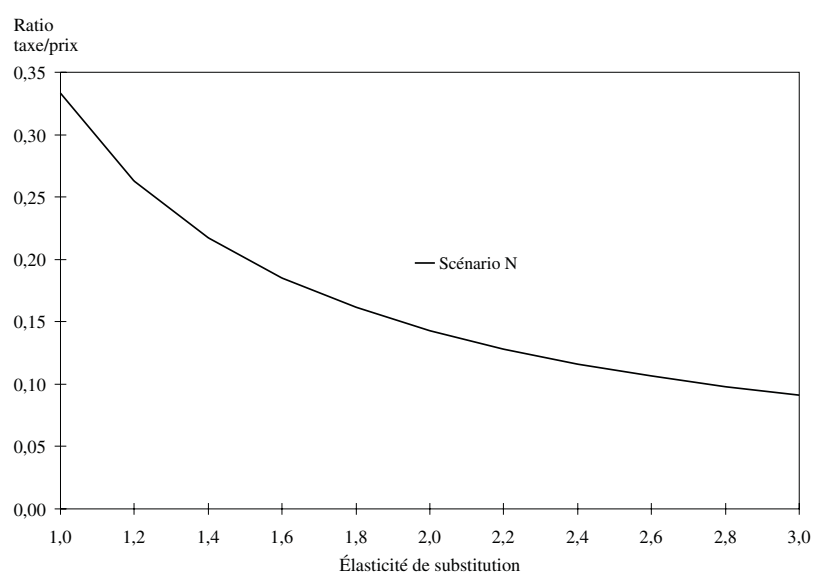

Négligeons les effets externes. Pour une forte élasticité, le prix HT diminue ainsi, entraînant une baisse plus rapide encore de la taxe, comme le suggère l'expression (21). La fiscalité prend donc une importance relative plus faible pour les fortes élasticités. Le prix TTC vaut

$$
p^{N}+t^{N}=\frac{\gamma(c(1+\lambda)+\eta)}{\gamma-1+\lambda(\gamma-2)}
$$

Le prix TTC est plus élevé que le prix TTC optimal au second rang, parce que la taxe renforce la stratégie de prix haut des producteurs. Dans ce contexte, la consommation est inférieure à ce que demande l'optimum

(26) $q^{N}=\left[\frac{\gamma(c(1+\lambda)+\eta}{\tau(\gamma-1+\lambda(\gamma-2))}\right]^{-\gamma}$

Nous comparons cette valeur avec $q^{\text {OSR }}$ dans la dernière section de la présente partie.

\section{Scénario F : l'Europe en position de force}

Dans ce scénario, un rôle proprement stratégique de la taxe est démontré. L'interaction se joue selon les règles suivantes :

- dans un premier temps, la Communauté fixe le niveau des taxes;

- dans un second temps, les producteurs fixent leur prix.

La différence avec le jeu simultané est importante dans le principe, mais aussi dans les conséquences sur l'équilibre comme les calculs le montrent. La seconde étape est une manière de modéliser l'hypothèse selon laquelle les producteurs doivent s'adapter aux conditions fiscales fixées par la Communauté. Bien sûr, la simple fixation d'une taxe représente l'exercice d'un pouvoir restreint, mais il est réel. D'autre part, l'étape 1 permet à la Communauté le calcul d'une exploitation optimale de la réaction (prévisible) des producteurs. C'est cet effet que nous appelons taxation stratégique.

\section{Niveau des prix et des taxes}

Ce type de problème se résout de manière rétrograde. Le choix des producteurs peut se poser dans les termes précédents et donc

(27) $p[t]=\frac{c \gamma+t}{\gamma-1}$

la taxe étant prise comme une donnée.

Pour la Communauté, l'équation (27) est incorporée dans son programme comme fonction de réaction. $\mathrm{La}$ Communauté choisit $t$ de manière à maximiser

(28) $U[q[p+t]]-(p+t) q+(1+\lambda) t q-\eta q$

sous la contrainte que $p=\frac{c \gamma+t}{\gamma-1}$. Cela donne

(29) $t^{F}=\frac{c\left(\lambda-\frac{1}{\gamma-1}\right)+\eta \gamma}{\gamma+\lambda(\gamma-1)+\frac{1}{\gamma-1}}$ 
Nous trouvons cette fois trois termes :

$\frac{\eta \gamma}{\gamma+\lambda(\gamma-1)+\frac{1}{\gamma-1}}$ représente ce qui reste de la motivation Pigouvienne de la taxation. Ce terme n'est toutefois jamais pur d'interaction avec la motivation stratégique, puisqu'il s'écarte systématiquement de $\eta$ y compris en l'absence de distorsion fiscale $(\lambda=0)$. Il se rapproche de l'idéal dans le cas où le coût des fonds publics est faible et où l'élasticité est très grande. En effet, une grande élasticité réduit les possibilités d'exploitation du pouvoir de marché par les producteurs, ce qui réduit donc aussi la nécessité de la contrer : seul subsiste à la limite l'effet externe ;

$\frac{c \gamma}{\gamma+\lambda(\gamma-1)+\frac{1}{\gamma-1}}$ mesure la taxation classique

(extraction de ressources pour le budget de l'État);

$\frac{-c}{(\gamma-1)+\left(\gamma+\lambda(\gamma-1)+\frac{1}{\gamma-1}\right)}$ est la motivation

stratégique. Cette contribution est négative : il s'agit d'exploiter la fonction de réaction des producteurs, qui amplifient les variations de la taxe par les variations des prix hors-taxe. En effet, on remarque que $\frac{\partial p}{\partial t}=\frac{1}{\gamma-1}$ et le prix TTC (réaction incluse) vaut

(30) $\frac{\gamma}{\gamma-1}\left(c+t^{\mathrm{F}}\right)$

D'où la consommation

$$
q^{F}=\left[\frac{\gamma^{2}(c(1+\lambda)+\eta)}{\tau(\gamma-1)\left(\gamma+\lambda(\gamma-1)+\frac{1}{\gamma-1}\right)}\right]^{-\gamma}
$$

Il est important de noter que le terme stratégique domine dès lors que les effets externes et le coût des fonds publics sont relativement faibles. Dans ce cas, la taxe est négative : il est nécessaire d'encourager la consommation. Cet effet paradoxal peut être compris de la manière suivante : avec une subvention, les consommateurs présentent alors une demande apparemment plus élastique, ce qui conduit nécessairement le monopole à la réduction du prix HT et du prix TTC.

\section{Sur ou sous-consommation?}

Nos analyses permettent de calculer prix, taxes et niveaux de productions dans des scénarios variés : - le scénario OSR représente le cas où l'économie maximise le surplus, la taxe remplissant aussi un rôle budgétaire. Nous donnons d'ailleurs un certain nombre de conditions (concurrence parfaite notamment) sous lesquelles ce résultat peut être atteint ;

- le scénario $\mathrm{N}$ suppose des moyens limités de la part de l'Europe dans ses tentatives d'influer sur le prix du gaz : elle ne dispose que de la taxe sur les importations et ne tente pas d'influer sur le cours hors-taxe ;

- dans le scénario F, l'Europe est plus forte et, bien qu'elle se limite encore à une taxe sur les importations, elle parvient à imposer ses choix fiscaux aux producteurs et exploite cette influence pour diriger le prix HT dans le sens qui lui est le plus favorable.

Nous trouvons notamment

(32) $q^{\mathrm{N}}<q^{\mathrm{F}}<q^{\mathrm{OSR}}$

Que penser donc de l'affirmation courante selon laquelle nous consommons trop d'énergie (assertion normative), alors que notre analyse montre que des protocoles d'accord inefficaces ( $N$ par exemple) conduisent plutôt à une sous-consommation? Il faut comprendre que tous nos scénarios prennent toujours en compte les effets environnementaux, ce qui n'est pas vraiment le cas des politiques réelles. En grossissant un peu le trait, nous pouvons penser qu'une politique réelle est de type $N$ où l'effet externe (représenté par $\eta$ ) serait négligé, donc les autorités publiques se comporteraient comme si $\eta$ valait zéro. Nous trouvons alors

$$
\left.q^{\mathrm{N}}\right|_{\eta=0}=\left[\frac{\gamma(1+\lambda) c}{\tau(\gamma-1+\lambda(\gamma-2))}\right]^{-\gamma}
$$

Or nous trouvons sur-consommation $\left.q^{N}\right|_{\eta=0}>q^{O S R}$ si et seulement si les effets externes sont assez grands (et donc si les négliger conduit à une surconsommation)

(34) $\eta>\frac{(1+\lambda)^{2} c}{\gamma-1+\lambda(\gamma-2)-1-\lambda}$

soit, dans une hypothèse assez optimiste sur l'élasticité $(\gamma=2$ et $\lambda=0,3), \eta>1,69 c$. S'il reste difficile de se prononcer de manière objective sur la valeur réelle de l'effet externe, on voit qu'il n'est pas nécessaire de croire à un effet externe énorme pour qu'on ait le sentiment que le scénario $N$ sans préoccupation environnementale conduise effectivement à la sur-consommation. 
Un calcul rapide permet de mesurer les enjeux. Le gaz naturel coûtait environ 150 euros par tep aux industriels en 1997 (IEA Statistics, 1998). Par ailleurs, les taxes suggérées par les études évaluant les moyens à mettre en œuvre afin de respecter le protocole de Kyoto varient de $\$ 30$ à $\$ 150$ par tonne de carbone (Bernard et Vielle, 2000), ce qui permet d'évaluer le coût par tep de \$24 à \$120 en appliquant le taux de conversion approprié. Si l'on interprète cette taxe comme un révélateur des effets externes, on voit que ces effets peuvent atteindre l'ordre de grandeur du prix TTC, ce qui les placent bien au-delà du coût de production. Le seuil de 1,69 c peut être largement dépassé par $\eta$, ce qui suggérerait, par un moyen très imparfait, que nous sommes effectivement dans un régime de sur-consommation.

\section{Le surplus : niveau et partage}

\section{Scénario N}

\section{Niveaux}

Calculons les surplus (l'indice $E$ vaut pour l'Europe, et l'indice $P$ pour les producteurs, $T$ indique le surplus total) :

(35) $S_{E}^{\mathrm{N}}=\frac{\tau(\gamma+\lambda(\gamma-1))}{\gamma(\gamma-1)}$

$$
\times\left[\frac{\gamma(c(1+\lambda)+\eta}{\tau(\gamma-1+\lambda(\gamma-2))}\right]^{1-\gamma}
$$

(36) $S_{P}^{N}=\frac{\tau}{\gamma}\left[\frac{\gamma(c(1+\lambda)+\eta}{\tau(\gamma-1+\lambda(\gamma-2))}\right]^{1-\gamma}$

d'où

$$
\text { (37) } \begin{aligned}
S_{T}^{N}= & \frac{\tau(2 \gamma-1+\lambda(\gamma-1))}{\gamma(\gamma-1)} \\
& \times\left[\frac{\gamma(c(1+\lambda)+\eta)}{\tau(\gamma-1+\lambda(\gamma-2))}\right]^{1-\gamma}
\end{aligned}
$$

Parts

Les parts de surplus sont maintenant calculables :

$$
\begin{aligned}
& \text { (38) } P_{E}^{\mathrm{N}}=\frac{\gamma+\lambda(\gamma-1)}{2 \gamma-1+\lambda(\gamma-1)} \\
& \text { (39) } P_{P}^{\mathrm{N}}=\frac{\gamma-1}{2 \gamma-1+\lambda(\gamma-1)}
\end{aligned}
$$

Graphique 2 : part des producteurs en fonction de $\gamma$ $\operatorname{avec} \lambda=0,33$

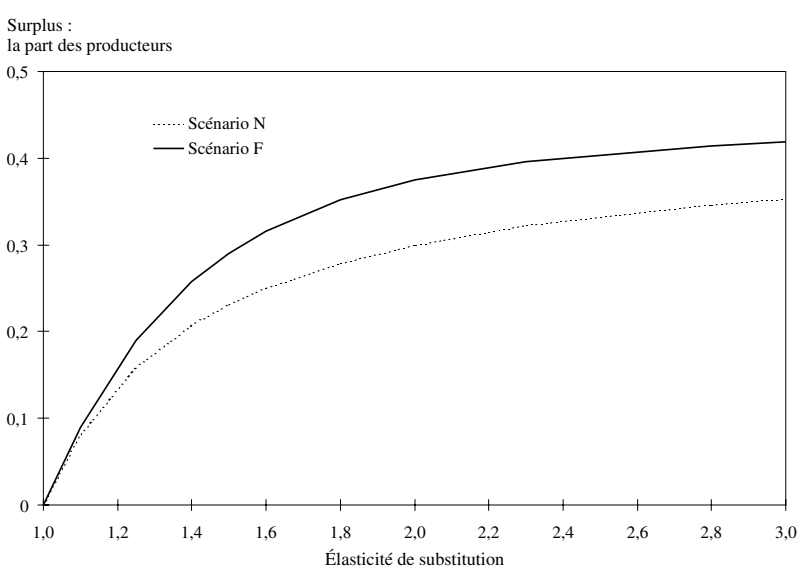

Graphique 3 : part des producteurs en fonction de $\lambda$ avec $\gamma=0,33$

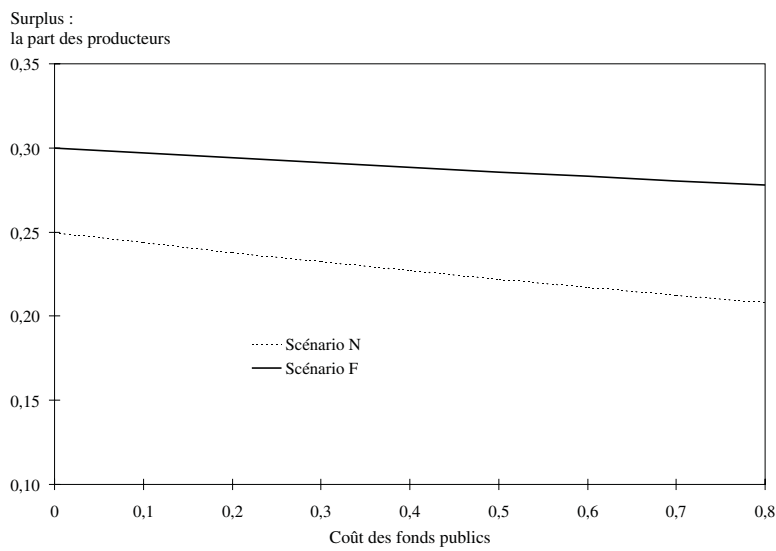

La part qui reste à l'Europe est toujours supérieure, quelle que soit la valeur de $\lambda$. Remarquons même que l'augmentation du coût des fonds publics est favorable : la part augmente et le surplus peut augmenter à certaines conditions. Ce résultat paradoxal doit être interprété correctement. Notre modélisation mesure le surplus engendré par le marché du gaz et ce surplus s'accroît lorsque les distorsions sont plus grandes ; cependant, les distorsions ont, sur la partie de l'économie que nous ne modélisons pas, un effet défavorable. Autrement dit, une situation fiscale défavorable ( $\lambda$ grand) n'est pas désirable en soit, mais elle permet de prendre une position (involontairement) plus sévère face aux producteurs, ce qui autorise une certaine compensation des inconvénients.

\section{Taux d'efficacité}

Il est intéressant de comparer le surplus engendré avec le surplus maximal (OSR). Nous calculons un taux d'efficacité en faisant le ratio des deux grandeurs, soit

$$
\text { (40) } \frac{S_{T}^{\mathrm{N}}}{S_{T}^{\text {OSR }}}=\frac{\gamma(2+\lambda)-1-\lambda}{\gamma(1+\lambda)-1-2 \lambda}\left[\frac{\gamma(1+\lambda)-1-2 \lambda}{\gamma(1+\lambda)-\lambda}\right]^{\gamma}
$$

Voir les graphiques 2 et 3 . 
Le graphique 4 montre les variations de ce taux. Il n'est jamais très élevé et l'efficacité est d'autant plus détériorée que l'élasticité de la demande est faible : l'exploitation fiscale et commerciale non concertée d'une élasticité faible conduit au désastre.

\section{Graphique 4 : taux d'efficacité dans le scénario $N$ en} fonction de $\gamma(\lambda=\mathbf{0 , 3 3})$

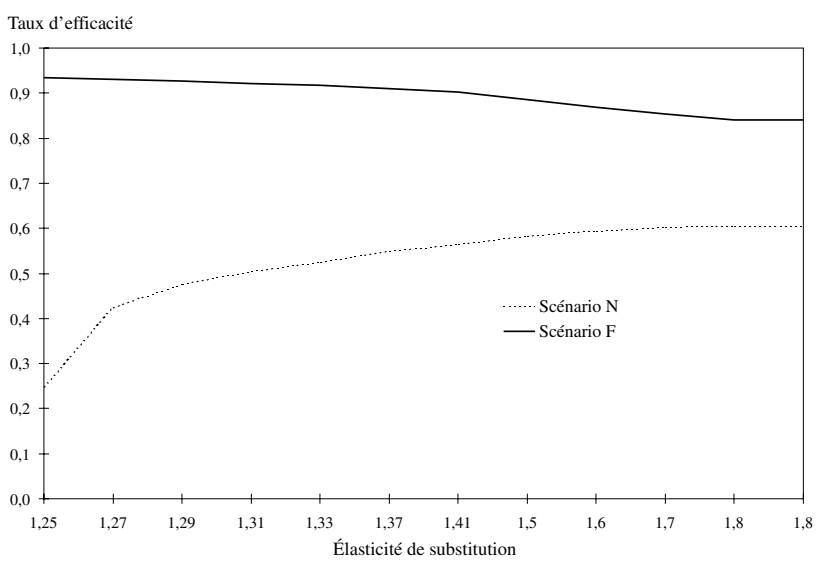

\section{Scénario F}

Niveaux

L'Europe obtient

$$
\text { (41) } \begin{aligned}
S_{E}^{\mathrm{F}}= & \frac{\left.\tau\left(\gamma^{2}(1+\lambda)-\gamma(1+2 \lambda)+1+\lambda\right)\right)}{\gamma^{2}(\gamma-1)} \\
& \times\left[\frac{\gamma(c(1+\lambda)+\eta)}{\tau(\gamma-1)\left(\gamma+\lambda(\gamma-1)+\frac{1}{\gamma-1}\right)}\right]^{1-\gamma}
\end{aligned}
$$

et les producteurs

$$
S_{P}^{\mathrm{F}}=\frac{\tau}{\gamma}\left[\frac{\gamma(c(1+\lambda)+\eta)}{\tau(\gamma-1)\left(\gamma+\lambda(\gamma-1)+\frac{1}{\gamma-1}\right)}\right]^{1-\gamma}
$$

Le surplus global vaut donc

$$
\begin{aligned}
S_{T}^{\mathrm{F}}= & \frac{\left.\tau\left(\gamma^{2}(2+\lambda)-2 \gamma(1+\lambda)+1+\lambda\right)\right)}{\gamma^{2}(\gamma-1)} \\
& \times\left[\frac{\gamma(c(1+\lambda)+\eta)}{\tau(\gamma-1)\left(\gamma+\lambda(\gamma-1)+\frac{1}{\gamma-1}\right)}\right]^{1-\gamma}
\end{aligned}
$$

Parts

Les parts revenant respectivement à l'Europe et aux producteurs sont donc

(44) $P_{E}^{\mathrm{F}}=\frac{\gamma+\lambda(\gamma-1)+\frac{1}{\gamma-1}}{2 \gamma+\lambda(\gamma-1)+\frac{1}{\gamma-1}}$

$$
\text { (45) } P_{P}^{\mathrm{F}}=\frac{\gamma}{2 \gamma+\lambda(\gamma-1)+\frac{1}{\gamma-1}}
$$

Taux d'efficacité

Le calcul donne

$$
\text { (46) } \begin{aligned}
\frac{S_{T}^{\mathrm{F}}}{S_{T}^{\mathrm{OSR}}}= & \frac{\gamma^{2}(2+\lambda)-2 \gamma(1+\lambda)+1+\lambda}{\gamma^{2}(1+\lambda)-\gamma(1+2 \lambda)+1+\lambda} \\
& \times\left[\frac{\gamma^{2}(1+\lambda)-\gamma(1+2 \lambda)+1+\lambda}{\gamma^{2}(1+\lambda)-\lambda \gamma}\right]
\end{aligned}
$$

Les variations en sont représentées sur le graphique 4. Les niveaux atteints sont toujours bien plus hauts que dans le scénario $N$. Les élasticités faibles voient le gain le plus significatif, puisque l'efficacité tend vers $100 \%$ lorsque $\gamma \mathrm{s}$ ' approche de 1 . Dans ce cas, la Communauté est capable de faire "simuler" par le marché une demande infiniment élastique, ce qui abolit le pouvoir de marché des producteurs.

\section{Effets des Directives sur le transport et la distribution}

A priori, les politiques communautaires sur le transport et la distribution du gaz affectent les coûts dans un sens favorable aux consommateurs. Dans une économie fermée, un progrès technique qui augmente l'efficacité technique ou une innovation réglementaire qui lamine les rentes sont des sources d'augmentation du surplus. Cette analyse n'est plus évidente dans notre contexte. En diminuant les coûts de transport et de distribution, il se pourrait qu'on augmente les possibilités d'abus de pouvoir de marché du producteur et que le surplus européen net s'en trouve diminué. Nous montrons qu'en fait le surplus produit est partagé entre la Communauté et les producteurs et nous calculons les parts.

Dans toutes les expressions de surplus (surplus partiel ou total, quel que soit le scénario) les surplus dépendent de la même manière des coûts dans l'économie, représentés par $c+\frac{\eta}{1+\lambda}$.

où

-le coût c représente le coût marginal de production; 
- le coût $\frac{\eta}{1+\lambda}$ représente le coût externe corrigé de l'effet double dividende (la taxation corrige l'effet externe et permet de réduire les taxes distorsives).

Plus ces coûts sont élevés et plus le surplus est faible. Plus précisément, les élasticités des surplus au coût sont identiques entre les scénarios et valent

(47) $-\frac{c}{S} \frac{\partial S}{\partial c}=\frac{c(\gamma-1)}{c+\frac{\eta}{1+\lambda}}$

Les effets externes modèrent les bénéfices, puisqu'une diminution de $c$ représente, en proportion, une moindre diminution du coût total. Une élasticité proche de 1 annule les bénéfices : dans ce cas, les producteurs augmentent considérablement leur prix lorsque leurs coûts diminuent, puisque leur marge unitaire augmente plus rapidement que le prix, et la demande diminue assez modérément.

Ainsi, pour une élasticité $\gamma=2$ et un effet externe négligeable, une baisse des coûts totaux de mise à disposition de $1 \%$ entraîne une hausse du surplus engendré par le gaz de $1 \%$. Les parts calculées dans les sections précédentes donnent une idée de la localisation de ce surplus additionnel.

Ce type de calcul a bien entendu des conséquences dans l'évaluation des politiques proconcurrentielles de la Communauté. Si la concurrence permet une diminution tendancielle des coûts de transport et de distribution, cette diminution peut être représentée par une diminution du coût $c$. Ainsi, si

(48) $c=c_{\text {Prod }}+c_{M D}$

(MD pour mise à disposition) nous trouvons la relation suivante

(49) $-\frac{c_{M D}}{S} \frac{\partial S}{\partial c}=\frac{c_{M D}(\gamma-1)}{c_{\text {Prod }}+c_{M D}+\frac{\eta}{1+\lambda}}$

Si les coûts de mise à disposition représentent la moitié des coûts totaux, alors le bénéfice en surplus de la diminution de ce coût de $1 \%$ est de $0,5 \%$ pour $\gamma=2$ et $\eta=0$ (hypothèses optimistes).

\section{Conclusion}

Un scénario dans lequel les producteurs seraient en position de force est évidemment défavorable à l'Europe. Nous n'en détaillons pas l'analyse, assez prévisible étant donné nos résultats. Tout renforcement de la position des producteurs (capacité à imposer le cadre de l'accord, usage d'instruments plus puissants) augmente leur rente et ne permet l'efficacité que s'ils utilisent des instruments complets.

Toute notre approche est focalisée sur l'offre, la demande n'étant jamais stratégique (elle est preneuse de prix). Notre étude permet de mettre en évidence de manière formelle l'intérêt qu'il y a à répondre stratégiquement à un comportement stratégique et permet de décrire l'ensemble des instruments (taxes et transferts) suffisant à l'établissement d'une plus grande efficacité. En résumé, le rôle de la fiscalité est de corriger les externalités budgétaires et environnementales et l'externalité "pécuniaire" (en fait un effet non concurrentiel).

La difficulté était de proposer un guide des comportements les plus favorables aux intérêts européens. Les solutions coopératives sont évidemment les meilleures, mais nous ne mésestimons pas la difficulté qu'il y a à s'accorder sur un niveau de partage donné. En particulier, il peut être tentant de retenir que la position européenne doit être raffermie. Cependant, les solutions extrêmes (tout le pouvoir de marchandage à l'Europe) ne sont pas nécessairement réalisables, avec la meilleure volonté, car il n'est pas certain que les producteurs adoptent une position subordonnée. La lutte d'influence sous-jacente est délicate à modéliser.

Pour mémoire, les politiques de quotas sont à exclure pour deux raisons. La première est qu'à quantité égale, il est préférable que l'État récupère des ressources grâce aux taxes plutôt que de laisser toutes les recettes entre les mains des producteurs. La seconde est qu'il n'y a guère de motif de protection de la production nationale.

Cette étude se focalise sur le gaz, mais deux énergies différentes peuvent recevoir des traitements fiscaux différents. Une raison simple est la différence entre effets externes : à cet égard le gaz peut recevoir un traitement plus favorable. Concernant la taxation à des fins de financement du budget, la demande de gaz étant considérée comme flexible, le gaz ne peut pas être considéré comme un réservoir fiscal très étendu et la fiscalité ne doit pas être particulièrement lourde.

D'une façon générale, la taxation stratégique de l'énergie doit tenir compte des effets de substituabilité/complémentarité entre différents produits énergétiques, tels qu'électricité, pétrole, 
gaz naturel, mais aussi énergies renouvelables. Dans un modèle "multiénergies", il faut spécifier non seulement une fonction d'utilité des consommateurs prenant en compte l'usage simultané de plusieurs énergies, mais aussi la structure de marché (monopole, concurrence parfaite ou oligopolistique) de chaque produit. De plus, certains producteurs peuvent être à l'intérieur de la Communauté, alors que d'autres sont localisés au-delà des frontières. Dans ce contexte, la taxation a un impact direct sur chaque bien, qui dépend de la réaction du producteur à la taxe, mais aussi un effet indirect. Ce dernier dépend du lien entre le prix TTC du bien $j$ et la taxe sur le bien $i$. L'intensité des effets directs et indirects dépend, d'une part, de la spécification des préférences des consommateurs et, d'autre part, du degré de pouvoir de marché et de la localisation des producteurs. Considérons par exemple le cas où deux sources d'énergies substituables sont consommées : une énergie est produite par un monopole externe à la l'Union Européenne, alors que l'autre est offerte par un producteur européen parfaitement concurrentiel. Le gouvernement a l'avantage stratégique et joue en premier. Cette approche, qui élargirait notre champ d'analyse, permettrait non seulement de calculer la taxe sur le bien produit par le monopole, mais aussi de montrer dans quelle mesure le bien concurrentiel peut servir de levier stratégique pour diminuer le pouvoir de marché du monopole et par conséquent s'il doit être taxé.

Les modèles classiques de description des prix des matières premières non renouvelables détenues par un monopole (telles que le gaz naturel et le pétrole) insistent sur l'évolution temporelle du prix. Hotelling (1931) a été à l'origine d'une vaste littérature montrant que l'évolution des prix doit suivre le taux d'intérêt ${ }^{(8)}$. Un résultat intéressant de cette voie de recherche est que si la production est concurrentielle, le schéma temporel est le même, le niveau des prix étant cependant plus bas que dans l'hypothèse d'un monopole. L'argument est fondé dans tous les cas sur l'arbitrage qui prendrait place si un autre sentier était suivi par l'économie. Un modèle entièrement satisfaisant devrait incorporer ces aspects dynamiques.

Par ailleurs, le modèle pourrait prendre en compte le commerce international de manière plus complète. En définitive, le gaz n'est jamais échangé contre de l'argent mais contre d'autres biens, présents ou futurs. Il serait donc assez naturel de supposer que la prospérité des producteurs de gaz produit un effet indirect positif sur la prospérité européenne. Cette version introduirait des motifs de coopération plus forts qui affecteraient également les politiques non coopératives.

\section{Notes}

(1) L'externalité ici est une externalité pure : il s'agit des effets secondaires de la consommation d'un individu qui se répercutent mécaniquement sur la collectivité. Il ne s'agit en rien d' «externalité pécuniaire » telle que le favoritisme envers une production communautaire. L'effet externe est donc négatif.

(2) Pour une analyse de la littérature sur la fiscalité environnementale notamment, voir Cremer et alii (2000).

(3) Cette forme réduite du coût des fonds publics, où $\lambda$ est exogène, est fréquente en théorie de la régulation (Laffont et Tirole, 1993).

(4) Nous n'étudions pas les taxes ad valorem dans cette étude. Notons que $t$ peut être interprétée comme une charge douanière.

(5) Nous ne ferons pas état de la littérature axiomatique sur la question, c'est-à-dire le «Nash bargaining » et ses développements.

(6) Voir cependant Jéhiel et Moldovanu (1995) pour une situation où la négociation n'aboutit à un résultat efficace qu'au bout d'un certain temps.

(7) En l'absence d'accord, l'Europe se tourne entièrement vers ses ressources propres, par exemple. La demande de ces ressources n'est pas modélisée.

(8) Il existe des versions un peu différentes dont nous ne parlerons pas ici. 


\section{Bibliographie}

Bernard A. et Vielle M. (2000). « Permis négociables et fiscalité », Economie Internationale, 82, 103-136.

Berndt R. et Wood D. (1975). « Technology, Prices, and the Derived Demand for Energy », Review of Economics and Statistics, 57, pp. 259-268.

Bishop R. (1968). " The Effects of Specific and ad Valorem Taxes », Quarterly Journal of Economics, 82, pp. 287-299.

Bohi D. R. et Toman M. A. (1996). The Economics of Energy Security, Kluwer Academic Publishers.

Commission des Communautés Européennes (1999). Energy in Europe Economic Foundations for Energy Policy.

Commission des Communautés Européennes (2000). Livre Vert Vers une stratégie européenne de la sécurité des approvisionnements.

Cremer H., Gahvari F. et Ladoux N. (2000). « La taxation des biens polluants ", Revue française d'économie, 14.

Goulder L. H. (1995). « Environmental Taxation and the Double Dividend: a Reader's Guide », International Tax and Public Finance, 2, pp. 157-183.

Griffin, J. A. (1977). « Interfuel Substitution Possibilities: a Translog Application to Intercountry Data », International Economic Review, 18, pp. 755-770.

Guesnerie R. et Laffont J.-J. (1978). « Taxing Price Makers ", Journal of Economic Theory, 19, pp. 423-455.

Guesnerie R. (1995). A Contribution to the Pure Theory of Taxation, Econometric Society Monographs, Cambridge University Press.

Hotelling H. (1931). " The Economics of Exhaustible Resources 》, Journal of Political Economy, 39, pp. 137-175.

IEA Statistics (1998). Energy Prices and Taxes, OCDE.

Jehiel P. et Moldovanu B. (1995). « Negative Externalities May Cause Delay in Negotiation ", Econometrica, 63, pp. 1321-35.

Laffont J.-J. (1987). Economie publique, Economica.

Laffont J.-J. et Tirole J. (1993). A Theory of Incentives in Procurement and Regulations, MIT Press.

Madlener R. (1996). « Econometric Analysis of Residential Energy Demand: A Survey ", The Journal of Energy Literature, 2, pp. 3-32.

Mathiesen L., Kjell R. et Thonstad K. (1987). « The European Natural Gas Market: Degrees of Market Power on the Selling Side », Document de Travail $n^{\circ} 22$, Central Bureau of Statistics, Norway.

Mirrlees J. A. (1976). « Optimal Tax Theory: A Synthesis », Journal of Public Economics, 6, pp. 327-358.

Myles G. D. (1987). « Tax Design in the Presence of Imperfect Competition: an Example ", Journal of Public Economics, 34, pp. 367-378.

Myles G. D. (1989). « Ramsey Tax Rules for Economies with Imperfect Competition ", Journal of Public Economics, 38, pp. 95-115.

Pearce D. W. (1991). " The Role of Carbon Taxes in Adjusting to Global Warming », Economic Journal, 101, pp. 938-948.

Reynou-Massant P. (1999). " Interfuel Competition in the Industrial Sector of seven OECD Countries », Energy Policy, 27, pp. 99-110.

Rubinstein A. (1982). « Perfect Equilibrium in a Bargaining Model », Econometrica, 50, pp. 97-109.
Sandmo A. (1975). "Optimal taxation in the Presence of Externalities ", Swedish Journal of Economics, 77, 86-98.

Seade J. (1985). " Profitable Cost Increase », Warwick Economic Research Paper $n^{\circ} 260$.

Tirole J. (1988). The Theory of Industrial Organization, Chapitre 11, MIT Press, Cambridge.

Wirl F. (1993). " Energy Pricing when Externalities are Taxed», Ressource and Energy Economics, 15, 255-270.

Woodland A. (1993). «A Micro-econometric Analysis of the Industrial Demand for Energy in NSW », Energy Journal, 14, pp. 57-89.

World Energy Assessment (2000). Energy and The Challenge of Sustainability. 